\title{
Tihonov theory and center manifolds for inhibitory mechanisms in enzyme kinetics.
}

\author{
A. M. Bersani ${ }^{1}$, A. Borri ${ }^{2}$, A. Milanesi ${ }^{3}$, P. Vellucci ${ }^{4}$ \\ ${ }^{1}$ Department of Mechanical and Aerospace Engineering, Sapienza University, \\ Roma,Italy \\ ${ }^{2}$ Institute for System Analysis and Computer Science "Antonio Ruberti" \\ (IASI-CNR), Roma,Italy \\ ${ }^{3}$ Department of Basic and Applied Sciences for Engineering, Sapienza University, \\ Roma,Italy \\ ${ }^{4}$ Department of Economics, Roma Tre University, Roma, Italy \\ *Email address for correspondence: pierluigi.vellucci@uniroma3.it \\ Communicated by Nicola Bellomo \\ Received on 01 12, 2017. Accepted on 04 20, 2017.
}

\begin{abstract}
In this paper we study the chemical reaction of inhibition, determine the appropriate parameter $\epsilon$ for the application of Tihonov's Theorem, compute explicitly the equations of the center manifold of the system and find sufficient conditions to guarantee that in the phase space the curves which relate the behavior of the complexes to the substrates by means of the tQSSA are asymptotically equivalent to the center manifold of the system. Some numerical results are discussed.

Keywords: Enzyme Kinetics, Inhibition, Tihonov's Theorem, Center Manifold, Perturbation Theory

AMS subject classification: 34D15, 34D20, 34E10, 92C45
\end{abstract}

\section{Introduction}

Let us consider a reaction where a substrate $\mathrm{S}$ binds reversibly to an enzyme $\mathrm{E}$ to form a complex $\mathrm{C}$. The complex can decay irreversibly to a product $\mathrm{P}$ and the enzyme, which is then free to bind another substrate molecule. This is summarized in the scheme

$$
E+S \underset{d}{\stackrel{a}{\rightleftharpoons}} C \stackrel{k}{\rightarrow} E+P
$$

where $a, d, k$ are kinetic parameters (supposed constant) associated with the reaction rates. For notational convenience we will use the same variable 
Bersani, Borri, Milanesi, Vellucci

names to denote both a chemical species and its concentration. For example, $E$ denotes both an enzyme and its concentration.

Assuming that the complex concentration is approximately constant after a short transient phase leads to the usual standard quasi-steady-state approximation (sQSSA)) [1], which is valid when the enzyme concentration is much lower than either the substrate concentration or the Michaelis constant $K_{M}=\frac{d+k}{a}$. The total QSSA (tQSSA) [2,3] is another approximation, and is valid for a broader range of parameter values covering both high and low enzyme concentrations.

The sQSSA and tQSSA are related to the asymptotic expansion of the solutions of the ODEs governing the process with respect to an appropriate parameter $[1,4-6]$; the topic can also be read in terms of center manifold, normal forms and bifurcation theory [7-9]. They are both developed setting the derivative of the intermediate complexes equal to zero. The theoretical justification for this assumption is given by Tihonov's Theorem 2.1 [10-13]. In Tihonov's framework, the assumption $\dot{C}_{i}=0$ is equivalent to impose the aforementioned "appropriate parameter" (the perturbation parameter, usually denoted by $\epsilon$ ) equal to zero (see, for example, [14,15].

In particular, Kumar and Josić [15] derive the mathematical expression of the center manifold for the tQSSA system just putting $\epsilon=0$. Although not seemingly strictly theoretically founded, this result remains as true for a wide class of systems (including the tQSSA model) and has a mathematical explanation, as we proved in a different paper [16]. It should be remarked that we still lack the theoretical investigation of the validity of the tQSSA in the case of successive reactions - where more parameters appear - but nevertheless it can be generalized to them. In particular, Theorem 2.1, as stated in [11], represents the generalization of Tihonov's Theorem [10,13] to the case of more parameters. The question is: what is the most appropriate parameter to develop an asymptotic expansion of the solutions of the ODEs modelling the reactions?

In a series of papers written by Palsson et al. [17] [18] [19] [20], the possibility to choose the required parameter has been related to the decoupling of the eigenvalues of the Jacobian matrix of the differential system. In [19] the authors focused on chains of reactions, obtaining a block form for the resulting Jacobian matrix.

In this paper we use the techniques shown in [19] in order to determine the appropriate parameter $\epsilon$ for the asymptotic expansions and give sufficient conditions to guarantee that in the phase space the curves which relate the behavior of the complexes to the substrates by means of the tQSSA are asymptotically equivalent to the center manifold of the system.

In Section 2 we recall the most important mathematical background 
Tihonov theory in inhibitory reactions.

concerning Tihonov's Theorem and Center Manifold Theory. In Section 3 we describe the mathematical model of the enzymatic inhibition reaction and, by means of an appropriate adimensionalization, we determine a suitable perturbation parameter $\epsilon$ which will be used to apply the tQSSA, in the framework of Tihonov's Theorem. In Section 4 we determine the Center Manifold of the system and discuss its asymptotic equivalence with the tQSSA. In Section 5 some perspectives of our research are discussed.

\section{Preliminary results and notations on nonlinear dynamical sys- tems.}

\subsection{Singular Perturbations}

For this section we will refer to the widespread book by W. Wasow [13], and - in particular - to its relevant section on Singular Perturbations. A systematic study of the qualitative aspects of such singular perturbation problems can be found in a series of papers by Tihonov [10-12], written in Russian.

According to [11], we consider $c+s$-dimensional differential systems of the form

$$
\begin{gathered}
\frac{d x}{d t}=f(x, y) \\
\epsilon \frac{d y}{d t}=g(x, y),
\end{gathered}
$$

where $x$ is $c$-dimensional and $y$ is $s$-dimensional. All variables are real, and $\epsilon$ is positive.

We assume that:

(A) The functions $f$ and $g$ in (2) are continuous in an open region $\Omega$ of the $(x, y)$-space.

(B) There is a $m$-dimensional vector function $\phi(x)$ continuous in $\xi_{1} \leq$ $x \leq \xi_{2}$ such that the points $(x, \phi(x))$, for all $\xi_{1} \leq x \leq \xi_{2}$, are in $\Omega$ and

$$
g(x, \phi(x)) \equiv 0 .
$$

(C) There exists a number $\eta>0$, independent of $x$, such that the relations

$$
\|y-\phi(x)\|<\eta, \quad y \neq \phi(x) \text { in } \xi_{1} \leq x \leq \xi_{2}
$$

imply

$$
g(x, y) \neq 0, \text { in } \xi_{1} \leq x \leq \xi_{2} .
$$


The function $\phi(x)$ will be referred to as a root of the equation $g(x, y)=0$. It is not excluded that $g(x, y)=0$ may have other roots besides $\phi(x)$. A root $\phi(x)$ that satisfies condition (C) will be called isolated in $\xi_{1} \leq x \leq \xi_{2}$.

Definition 2.1. The system of differential equations

$$
\epsilon \frac{d y}{d t}=g(x, y)
$$

in which $x$ is a parameter, will be called the boundary layer equation belonging to the system (2). We also assume:

(D) The singular point $y=\phi(x)$ of the boundary layer equation (3) is asymptotically stable for all $\xi_{1} \leq x \leq \xi_{2}$.

The root $\phi(x)$ will be called, briefly, a stable root in $\xi_{1} \leq x \leq \xi_{2}$, if assumption (D) is satisfied.

In accordance with our previous terminology we refer to the problem consisting of the system (2) together with the initial condition

$$
x=\alpha, \quad y=\beta, \text { for } t=0
$$

as the full problem. The reduced problem is here defined by

$$
\begin{gathered}
\frac{d x}{d t}=f(x, \phi(x)) \\
y=\phi(x), \\
x=\alpha, \quad \text { for } t=0
\end{gathered}
$$

The system (5) is, of course, obtained by setting $\epsilon=0$ in (2) and choosing the particular root $y=\phi(x)$ of the equation $g(x, y)=0$. Moreover, we assume:

(E) The full, as well as the reduced problem has a unique solution in an interval $0 \leq t \leq T$.

(F) The asymptotic stability of the singular point $y=\phi(x)$ is uniform with respect to $x$ in $\xi_{1} \leq x \leq \xi_{2}$.

For a convenient formulation of Tihonov's Theorem, according to [13], we introduce one more term.

Definition 2.2. A point $(\alpha, \beta) \in \Omega, \xi_{1} \leq \alpha \leq \xi_{2}$ is said to lie in the domain of influence of the stable root $y=\phi(x)$ if the solution of the problem

$$
d y / d \tau=g(\alpha, y), \quad y(0)=\beta
$$

exists and remains in $\Omega$ for all $\tau>0$, and if it tends to $\phi(\alpha)$, as $\tau \rightarrow+\infty$. 


\section{Tihonov theory in inhibitory reactions.}

Theorem 2.1. Let Assumptions (A) to $(F)$ be satisfied and let $(\alpha, \beta)$ be a point in the domain of influence of the root $y=\phi(x)$. Then the solution $x(t, \epsilon), y(t, \epsilon)$ of the full initial value problem (2), (4) is connected with the solution $x_{0}(t), y_{0}(t)=\phi\left(x_{0}(t)\right)$ of the reduced problem (5), (6) by the limit relations

$$
\begin{aligned}
& \lim _{\epsilon \rightarrow 0} x(t, \epsilon)=x_{0}(t), \quad 0 \leq t \leq T_{0} \\
& \lim _{\epsilon \rightarrow 0} y(t, \epsilon)=y_{0}(t)=\phi\left(x_{0}(t)\right) \quad 0<t \leq T_{0}
\end{aligned}
$$

Here $T_{0}$ is any number such that $y=\phi\left(x_{0}(t)\right)$ is an isolated stable root of $g\left(x_{o}(t), y\right)=0$ for $0 \leq t \leq T_{0}$. The convergence is uniform in $0 \leq t \leq T_{0}$, for $x(t, \epsilon)$, and in any interval $0<t_{1} \leq t \leq T_{0}$ for $y(t, \epsilon)$.

Tihonov's Theorem 2.1 is only the first step in the asymptotic solution of initial value problems of the singular perturbation type. The most natural approach to these problems is to attempt a solution in the form of a series in powers of $\epsilon$ :

$$
x=\sum_{r=0}^{\infty} x_{r}(t) \epsilon^{r}, \quad y=\sum_{r=0}^{\infty} y_{r}(t) \epsilon^{r}
$$

and to determine the coefficients $x_{r}(t), y_{r}(t)$ by means of formal substitution and comparison of coefficients.

It is clear that we have to relate the series (8) to the behavior of the solution of (2) in the boundary layer. For values of $t$ that are small of order $O(\epsilon)$ the solution to our perturbation problem can be found by means of the stretching transformation $t=\tau \epsilon$. Hence, the stretched, or inner, form of the original problem is

$$
\begin{gathered}
\frac{d x}{d \tau}=\epsilon f(x, y), \quad \frac{d y}{d \tau}=g(x, y), \\
x=\alpha, \quad y=\beta, \quad \text { for } \tau=0 .
\end{gathered}
$$

If the functions $f$ and $g$ are analytic the solutions of this problem have convergent expansions in powers of $\epsilon$, say,

$$
x(\tau, \epsilon)=\sum_{r=0}^{\infty} u_{r}(\tau) \epsilon^{r}, \quad y(\tau, \epsilon)=\sum_{r=0}^{\infty} v_{r}(\tau) \epsilon^{r},
$$

and the series reduce to $x=\alpha, y=\beta$, identically in $\epsilon$, for $\tau=0$, so that

$$
\begin{aligned}
& u_{0}(0)=\alpha, \quad v_{0}(0)=\beta, \\
& u_{r}(0)=0, \quad v_{r}(0)=0, \quad r>0 .
\end{aligned}
$$


If $f$ and $g$ are only assumed to be infinitely differentiable it is possible to prove that the series (10) calculated by the same formal operations as in the analytic case represent the solution asymptotically, as $\epsilon \rightarrow 0^{+}$, in every finite $\tau$-interval.

In this paper we consider, as in [11], the case of several parameters $\left\{\epsilon_{j}\right\}_{j=1}^{m}$ of the same order, namely, $\epsilon_{j}=\rho_{j-1} \epsilon$ for each $j=1, \ldots, m$ and $\rho_{j}>0\left(\rho_{0}=1\right)$. We obtain a system of the form

$$
\begin{aligned}
\frac{d x}{d t} & =f\left(x, y_{1}, \ldots, y_{m} ; t\right), \\
\epsilon \frac{d y_{j}}{d t} & =\frac{1}{\rho_{j-1}} g_{j}\left(x, y_{1}, \ldots, y_{m} ; t\right), \quad j=1,2, \ldots, m,
\end{aligned}
$$

which becomes, in the vectorial form,

$$
\begin{aligned}
\frac{d x}{d t} & =f(x, y ; t), \\
\epsilon \frac{d y}{d t} & =g(x, y ; t),
\end{aligned}
$$

where

$$
y=\left(y_{1}, \ldots, y_{m}\right)^{t}, \quad g=\left(g_{1}, \frac{1}{\rho_{1}} g_{2}, \ldots, \frac{1}{\rho_{m-1}} g_{m}\right)^{t} .
$$

Then it is possible to apply, for the system (13), Tihonov's Theorem.

\subsection{Center Manifold}

Let us consider vector fields of the following form

$$
\begin{aligned}
& \dot{x}=A x+f(x, y), \\
& \dot{y}=B y+g(x, y), \quad(x, y) \in \mathbb{R}^{c} \times \mathbb{R}^{s},
\end{aligned}
$$

where

$$
\begin{aligned}
& f(0,0)=0, \quad D f(0,0)=0, \\
& g(0,0)=0, \quad D g(0,0)=0 .
\end{aligned}
$$

where $A$ is a $c \times c$ matrix having eigenvalues with zero real parts, $B$ is an $s \times s$ matrix having eigenvalues with negative real parts, $f$ and $g$ are $C^{r}$ functions $(r \geq 2)$ and $D$ is the jacobian matrix of the system.

Definition 2.3 (Center Manifold [7-9,21]). An invariant manifold will be called a center manifold for (14) if it can locally be represented as follows

$$
W^{c}(0)=\left\{(x, y) \in \mathbb{R}^{c} \times \mathbb{R}^{s}|y=h(x),| x \mid<\delta, h(0)=0, D h(0)=0\right\}
$$

for $\delta$ sufficiently small. 


\section{Tihonov theory in inhibitory reactions.}

To find a center manifold, all we need do is to solve $[8,9]$ :

$$
\mathcal{N}(h(x)) \equiv D h(x)(A x+f(x, h(x)))-B h(x)-g(x, h(x))=0,
$$

and the following theorem gives us a method for computing an approximate solution of (16) to any desired degree of accuracy. Let us remark [8,9,21] the non uniqueness of the center manifold.

Theorem 2.2 (Approximation [7-9]). Let $\phi: \mathbb{R}^{c} \rightarrow \mathbb{R}^{s}$ be a $C^{1}$ mapping with $\phi(0)=D \phi(0)=0$ such that $\mathcal{N}(\phi(x))=O\left(|x|^{q}\right)$ as $x \rightarrow 0$ for some $q>1$. Then

$$
|h(x)-\phi(x)|=O\left(|x|^{q}\right), \quad \text { as } x \rightarrow 0 .
$$

When we consider a system depending on a parameter $\epsilon$

$$
\begin{aligned}
& \dot{x}=A x+f(x, y, \epsilon), \\
& \dot{y}=B y+g(x, y, \epsilon), \quad(x, y, \epsilon) \in \mathbb{R}^{c} \times \mathbb{R}^{s} \times \mathbb{R},
\end{aligned}
$$

where

$$
\begin{aligned}
& f(0,0,0)=0, \quad D f(0,0,0)=0, \\
& g(0,0,0)=0, \quad D g(0,0,0)=0,
\end{aligned}
$$

following $[8,9,21]$, we can handle these parameterized systems treating the parameter $\epsilon$ as a new dependent variable as follows.

$$
\begin{aligned}
& \dot{x}=A x+f(x, y, \varepsilon), \\
& \dot{\varepsilon}=0, \\
& \dot{y}=B y+g(x, y, \varepsilon), \quad(x, y, \epsilon) \in \mathbb{R}^{c} \times \mathbb{R}^{s} \times \mathbb{R} .
\end{aligned}
$$

This system has a fixed point at $(x, y, \epsilon)=(0,0,0)$. The matrix associated with the linearization of (19) about this fixed point has $c+1$ eigenvalues with zero real part and $s$ eigenvalues with negative real part. Let us now apply center manifold theory. Modifying definition 2.3, a center manifold will be represented as a graph over the $x$ and $\varepsilon$ variables, i.e., the graph of $h(x, \varepsilon)$ for $x$ and $\varepsilon$ sufficiently small. Theorem 2.2 still applies, with the vector field reduced to the center manifold given by

$$
\begin{aligned}
& \dot{u}=A u+f(u, h(u, \varepsilon), \varepsilon), \\
& \dot{\varepsilon}=0, \quad(u, \varepsilon) \in \mathbb{R}^{c} \times \mathbb{R} .
\end{aligned}
$$




\section{Mathematical model of the inhibition mechanism}

An inhibition reaction, where an inhibitor $S_{2}$ competes with a substrate $S_{1}$ for the same enzyme $E$, is summarized by the following scheme

$$
\left\{\begin{array}{l}
E+S_{1} \underset{d_{1}}{\stackrel{a_{1}}{\rightleftharpoons}} C_{1} \stackrel{k_{1}}{\longrightarrow} E+P_{1} \\
E+S_{2} \underset{d_{2}}{\stackrel{a_{2}}{\rightleftharpoons}} C_{2} \stackrel{k_{2}}{\longrightarrow} E+P_{2}
\end{array}\right.
$$

where $P_{1}, P_{2}$ are the reaction products and $C_{1}, C_{2}$ the enzyme-substrate complexes. In this kind of reaction, it is clear that $S_{1}$ and $S_{2}$ have the same role of reciprocal inhibitors ( $S_{1}$ inhibits $S_{2}$ as $S_{2}$ inhibits $S_{1}$ ). Introducing the total substrates

$$
\overline{S_{1}}=S_{1}+C_{1}, \overline{S_{2}}=S_{2}+C_{2},
$$

by conservation laws we have

$$
\overline{S_{1}}+P_{1}=S_{1 T}, \overline{S_{2}}+P_{2}=S_{2 T}, E+C_{1}+C_{2}=E_{T} .
$$

Then we can write the following Cauchy Problem:

$$
\left\{\begin{array}{l}
\frac{d \overline{S_{1}}}{d t}=-k_{1} C_{1} \\
\frac{d C_{1}}{d t}=a_{1}\left[\left(\overline{S_{1}}-C_{1}\right)\left(E_{T}-C_{1}-C_{2}\right)-K_{1 M} C_{1}\right] \\
\frac{d S_{2}}{d t}=-k_{2} C_{2} \\
\frac{d C_{2}}{d t}=a_{2}\left[\left(\overline{S_{2}}-C_{2}\right)\left(E_{T}-C_{1}-C_{2}\right)-K_{2 M} C_{2}\right] \\
\overline{S_{1}}(0)=S_{1 T} \quad ; \quad \overline{S_{2}}(0)=S_{2 T} \quad ; \quad C_{1}(0)=C_{2}(0)=0,
\end{array}\right.
$$

where

$$
K_{1 M}=\frac{d_{1}+k_{1}}{a_{1}}, \quad K_{2 M}=\frac{d_{2}+k_{2}}{a_{2}}
$$

are the Michaelis constants. It is easy to see that in this case the origin $X^{*}:=\left({\overline{S_{1}}}^{*}, C_{1}^{*},{\overline{S_{2}}}^{*}, C_{2}\right)=(0,0,0,0)$ is the only fixed point of the system. The linear approximation of the system around $X^{*}$ is represented by the following system:

$$
\left\{\begin{array}{l}
\frac{d \overline{S_{1}}}{d t}=-k_{1} C_{1} \\
\frac{d C_{1}}{d t}=a_{1}\left[E_{T} \overline{S_{1}}-E_{T} C_{1}-K_{1 M} C_{1}\right] \\
\frac{d \overline{S_{2}}}{d t}=-k_{2} C_{2} \\
\frac{d C_{2}}{d t}=a_{2}\left[E_{T} \overline{S_{2}}-E_{T} C_{2}-K_{2 M} C_{2}\right]
\end{array},\right.
$$




\section{Tihonov theory in inhibitory reactions.}

with Jacobian at the origin

$$
D(0,0,0,0)=\left(\begin{array}{cccc}
0 & -k_{1} & 0 & 0 \\
a_{1} E_{T}-a_{1}\left(E_{T}+K_{1 M}\right) & 0 & 0 \\
0 & 0 & 0 & -k_{2} \\
0 & 0 & a_{2} E_{T}-a_{2}\left(E_{T}+K_{2 M}\right)
\end{array}\right),
$$

with two blocks. An easy computation shows that

$$
\begin{aligned}
|D-\lambda \mathbb{I}| & =\left[\lambda^{2}+a_{1}\left(E_{T}+K_{1 M}\right) \lambda+k_{1} a_{1} E_{T}\right]\left[\lambda^{2}+\right. \\
& \left.+a_{1}\left(E_{T}+K_{2 M}\right) \lambda+k_{2} a_{2} E_{T}\right]
\end{aligned}
$$

then the eigenvalues are

$$
\begin{aligned}
\left(\lambda_{ \pm}\right)_{i}= & \frac{-a_{i}\left(E_{T}+K_{i M}\right) \pm \sqrt{a_{i}^{2}\left(E_{T}+K_{i M}\right)^{2}-4 a_{i} k_{i} E_{T}}}{2}= \\
& \frac{a_{i}\left(E_{T}+K_{i M}\right)}{2}\left(-1 \pm \sqrt{1-\frac{4 K_{i} E_{T}}{\left(E_{T}+K_{i M}\right)^{2}}}\right)
\end{aligned}
$$

where we have denoted the Van Slyke-Cullen constants by $K_{i}=\frac{k_{i}}{a_{i}}, i=1,2$. Now it is clear that

$$
\frac{4 K_{i} E_{T}}{E_{T}+K_{i M}} \ll 1 \Longrightarrow\left(\lambda_{-}\right)_{i} \ll\left(\lambda_{+}\right)_{i},
$$

$i=1,2$, i.e., according to Palsson [19], we have a sufficient condition for the separation of time scales.

We could choose

$$
\tilde{\epsilon}_{i}:=\frac{K_{i} E_{T}}{\left(E_{T}+K_{i M}\right)^{2}},
$$

as perturbation parameter. It easy to show that $\tilde{\epsilon}_{i}<\frac{1}{4}$ for every choice of data. However, in order to follow the paper [6], it is more convenient to take

$$
\epsilon=\max \left\{\epsilon_{i}:=\frac{K_{i} E_{T}}{\left(E_{T}+K_{i M}+S_{i T}\right)^{2}} ; \quad i=1,2\right\}<\frac{1}{4}
$$

as perturbation parameter (note that $\epsilon_{i}<\tilde{\epsilon}_{i}$ ). Furthermore, thanks to the symmetry of the problem, we can suppose that $\epsilon=\epsilon_{1}$. With this choice the following change of variables

$$
\overline{S_{i}}=\alpha_{i} s_{i}, \quad C_{i}=\beta_{i} c_{i}, \quad t=\gamma \tau,
$$


with

$$
\alpha_{i}=S_{i T}, \quad \beta_{i}=\frac{E_{T} S_{i T}}{E_{T}+K_{i M}+S_{i M}}, \quad \gamma=\frac{1}{a_{1}\left(E_{T}+K_{1 M}+S_{1 M}\right)},
$$

$i=1,2$, provides an adimensionalization of the model equations (see [6]):

$$
\left\{\begin{aligned}
\frac{\alpha_{1}}{\gamma} \frac{d \overline{s_{1}}}{d \tau} & =-k_{1} \beta_{1} c_{1} \\
\frac{\beta_{1}}{\gamma} \frac{d c_{1}}{d \tau} & =a_{1}\left[\beta_{1}^{2} c_{1}^{2}-\left(E_{T}+\alpha_{1} \overline{s_{1}}-\beta_{2} c_{2}+K_{1 M}\right) \beta_{1} c_{1}+\right. \\
& \left.-\alpha_{1} \overline{s_{1}} \beta_{2} c_{2}+\alpha_{1} \overline{s_{1}} E_{T}\right] \\
\frac{\alpha_{2}}{\gamma} \frac{d \overline{s_{2}}}{d \tau} & =-k_{2} \beta_{2} c_{2} \\
\frac{\beta_{2}}{\gamma} \frac{d c_{2}}{d \tau} & =a_{2}\left[\beta_{2}^{2} c_{2}^{2}-\left(E_{T}+\alpha_{2} \overline{s_{2}}-\beta_{1} c_{1}+K_{2 M}\right) \beta_{2} c_{2}+\right. \\
& \left.-\alpha_{2} \overline{s_{2}} \beta_{1} c_{1}+\alpha_{2} \overline{s_{2}} E_{T}\right]
\end{aligned}\right.
$$

thanks to which we obtain the system of equations for the inner solutions:

$$
\left\{\begin{aligned}
\frac{d \overline{s_{1}}}{d \tau} & =-\epsilon c_{1} \\
\frac{d c_{1}}{d \tau} & =\sigma_{1} \eta_{1} c_{1}^{2}-\left(\eta_{1}+\kappa_{1 M}\right) c_{1}-\sigma_{1} \overline{s_{1}} c_{1}+\sigma_{2} \eta_{1} c_{1} c_{2}+\overline{s_{1}}-\sigma_{2} c_{2} \overline{s_{1}} \\
\frac{d \overline{s 2}}{d \tau} & =-\epsilon \overline{k_{2} \eta_{2}} c_{2} \\
\frac{d c_{2}}{d \tau} & =\frac{a_{2}}{a_{1}}\left[\eta_{1} \sigma_{2} c_{2}^{2}-\left(\eta_{1}+\frac{K_{2 M}}{K_{1 M}} \kappa_{1 M}\right) c_{2}+\right. \\
& \left.+\left(\eta_{1} \sigma_{1} c_{1}-\frac{S_{2 T}}{S_{1 T}} \sigma_{1} \overline{s_{2}}\right) c_{2}+\frac{\eta_{1}}{\eta_{2}} \overline{s_{2}}-\frac{\sigma_{1} \eta_{1}}{\eta_{2}} \overline{s_{2}} c_{1}\right]
\end{aligned}\right.
$$

where

$$
\sigma_{i}=\frac{S_{i T}}{E_{T}+K_{i T}+S_{i T}} ; \quad \eta_{i}=\frac{E_{T}}{E_{T}+K_{i T}+S_{i T}} ; \quad \kappa_{i M}=\frac{K_{i M}}{E_{T}+K_{i T}+S_{i T}}
$$

$i=1,2$ (note that $\sigma_{i}+\eta_{i}+\kappa_{i M}=1$ ). We now write the system of equations that gives the outer solutions. To this aim we set

$$
\bar{\gamma}:=\frac{1}{k_{1} \eta_{1}}
$$

and note that putting $T=\frac{t}{\gamma}$, we see that

$$
T=\frac{\gamma}{\bar{\gamma}} \tau=\epsilon \tau
$$

So we obtain

$$
\left\{\begin{aligned}
\frac{d \overline{s_{1}}}{d T} & =-c_{1} \\
\epsilon \frac{d c_{1}}{d T} & =\sigma_{1} \eta_{1} c_{1}^{2}-\left(\eta_{1}+\kappa_{1 M}\right) c_{1}-\sigma_{1} \overline{s_{1}} c_{1}+\sigma_{2} \eta_{1} c_{1} c_{2}+\overline{s_{1}}-\sigma_{2} c_{2} \overline{s_{1}} \\
\frac{d \bar{s}_{2}}{d T} & =-\frac{k_{2} \eta_{2}}{k_{1} \eta_{1}} c_{2} \\
\epsilon \frac{d c_{2}}{d T} & =\frac{a_{2}}{a_{1}}\left[\eta_{1} \sigma_{2} c_{2}^{2}-\left(\eta_{1}+\frac{K_{2 M}}{K_{1 M}} \kappa_{1 M}\right) c_{2}+\right. \\
& \left.+\left(\eta_{1} \sigma_{1} c_{1}-\frac{S_{2 T}}{S_{1 T}} \sigma_{1} \overline{s_{2}}\right) c_{2}+\frac{\eta_{1}}{\eta_{2}} \overline{s_{2}}-\frac{\sigma_{1} \eta_{1}}{\eta_{2}} \overline{s_{2}} c_{1}\right] .
\end{aligned}\right.
$$




\section{Tihonov theory in inhibitory reactions.}

At this point we can apply Tihonov's Theorem. Putting $\epsilon=0$, we obtain the reduced system of equations, a system which is both of differential and algebraic type:

$$
\left\{\begin{array}{l}
\frac{d \overline{s_{1}}}{d T}=-c_{1} \\
\sigma_{1} \eta_{1} c_{1}^{2}-\left(\eta_{1}+\kappa_{1 M}\right) c_{1}-\sigma_{2} c_{2} \overline{s_{1}}-\sigma_{1} \overline{s_{1}} c_{1}+\sigma_{2} \eta_{1} c_{1} c_{2}+\overline{s_{1}}=0 \\
\frac{d \overline{s_{2}}}{d T}=-\frac{k_{2} \eta_{2}}{k_{1} \eta_{1}} c_{2} \\
\eta_{1} \sigma_{2} c_{2}^{2}-\left(\eta_{1}+\frac{K_{2 M}}{K_{1 M}} \kappa_{1 M}\right) c_{2}-\frac{\sigma_{1} \eta_{1}}{\eta_{2}} \overline{s_{2}} c_{1}+ \\
+\left(\eta_{1} \sigma_{1} c_{1}-\frac{S_{2 T}}{S_{1 T}} \sigma_{1} \overline{s_{2}}\right) c_{2}+\frac{\eta_{1}}{\eta_{2}} \overline{s_{2}}=0
\end{array}\right.
$$

From the algebraic equations we can obtain cumbersome expressions for $c_{1}\left(\overline{s_{1}}, \overline{s_{2}}\right)$ and $c_{2}\left(\overline{s_{1}}, \overline{s_{2}}\right)$, which are useful for the numerical integration of the differential equations.

For the sake of clarity we prefer here to show the reverse relations:

$$
\begin{gathered}
\overline{s_{1}}=\frac{\sigma_{1} \eta_{1} c_{1}^{2}-\left(\eta_{1}+\kappa_{1 M}\right) c_{1}+\sigma_{2} \eta_{1} c_{1} c_{2}}{\left(\sigma_{1} c_{1}-\sigma_{2} c_{2}-1\right)} \\
\overline{s_{2}}=\frac{\eta_{1} \sigma_{2} c_{2}^{2}-\left(\eta_{1}+\frac{K_{2 M}}{K_{1 M}} \kappa_{1 M}\right) c_{2}+\eta_{1} \sigma_{1} c_{1} c_{2}}{\left(\frac{S_{2 T}}{S_{1 T}} \sigma_{1} c_{2}-\frac{\eta_{1}}{\eta_{2}} \sigma_{1} c_{1}-\frac{\eta_{1}}{\eta_{2}}\right)} .
\end{gathered}
$$

Figure 1 compares the time behavior of the complexes (left) and of the substrates (right), respectively, obtained as numerical solutions of the system (35) and of their tQSSA (36), in a case where the parameter values are chosen is such a way to obtain a small value of the perturbation parameter $(\epsilon=0.02)$.

Figure 2 compares the time behavior of the complexes (left) and of the substrates (right), respectively, obtained as numerical solutions of the system (35) and of their tQSSA (36), in a case where the parameter values are chosen is such a way to obtain a high value of the perturbation parameter $(\epsilon=0.23)$, very close to its upper bound.

The figures show a very good approximation, even in the stressed case, in accordance with Tihonov's Theorem. 
Bersani, Borri, Milanesi, Vellucci
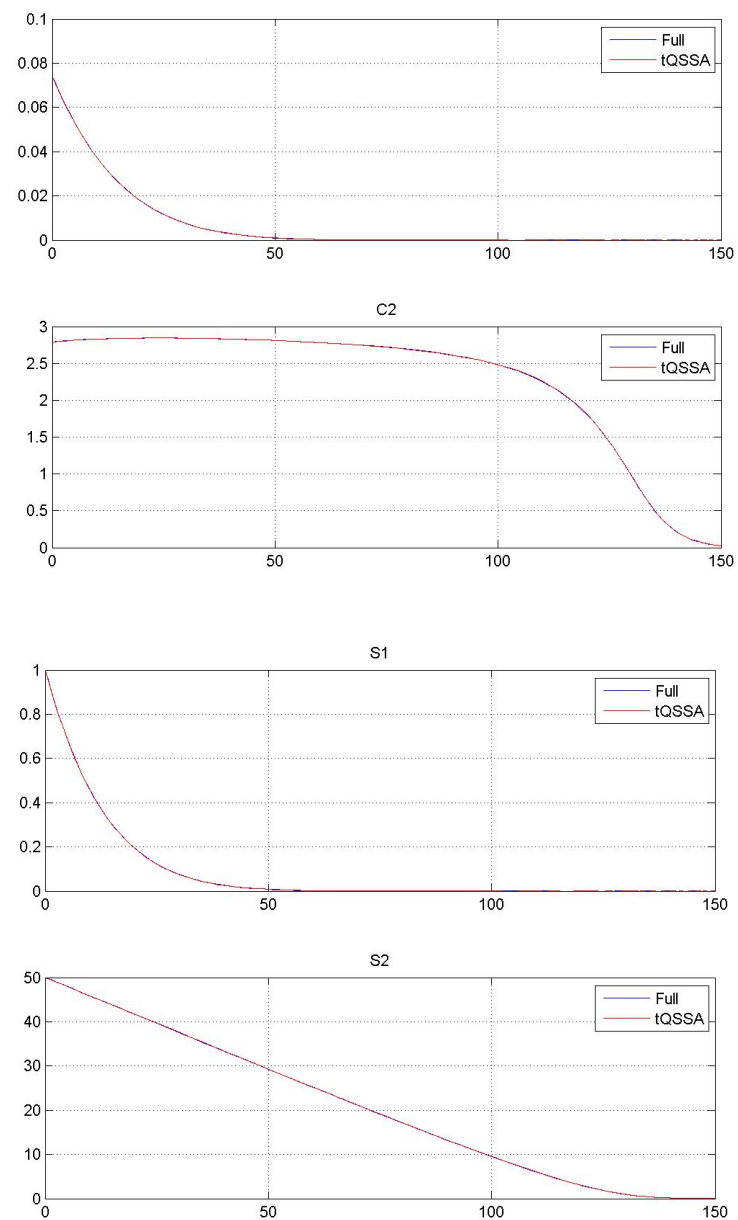

Figure 1. Comparison of the complexes (left) and of the substrates (right), solution of the system (35), with their tQSSA (36). The parameter set is the following: for the first reaction $a_{1}=k_{1}=1 ; d_{1}=0.1 ; E_{T}=0.1 ; S_{1} T=1 ; K_{1} M=1.1 ; K=1 ; \epsilon_{1}=0.02$; for the second reaction $a_{2}=0.1 ; k_{2}=10 ; d_{2}=0.01 ; E_{T}=0.1 ; S_{2} T=50 ; K_{2} M=100.1 ; K=$ $100 ; \epsilon_{2}=0.00044$. Thus $\epsilon=0.02$. 
Tihonov theory in inhibitory reactions.

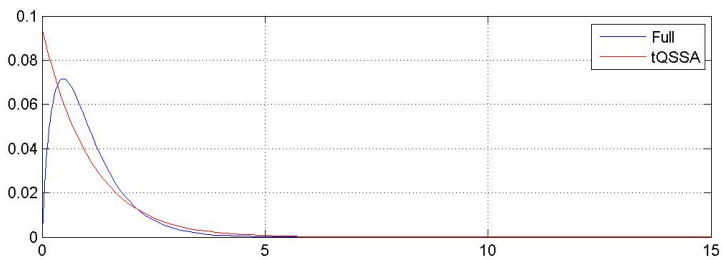

$\mathrm{C2}$

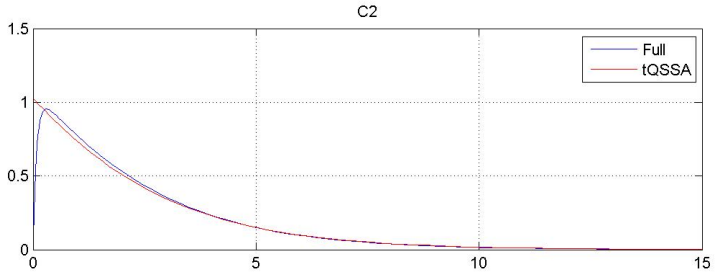

st
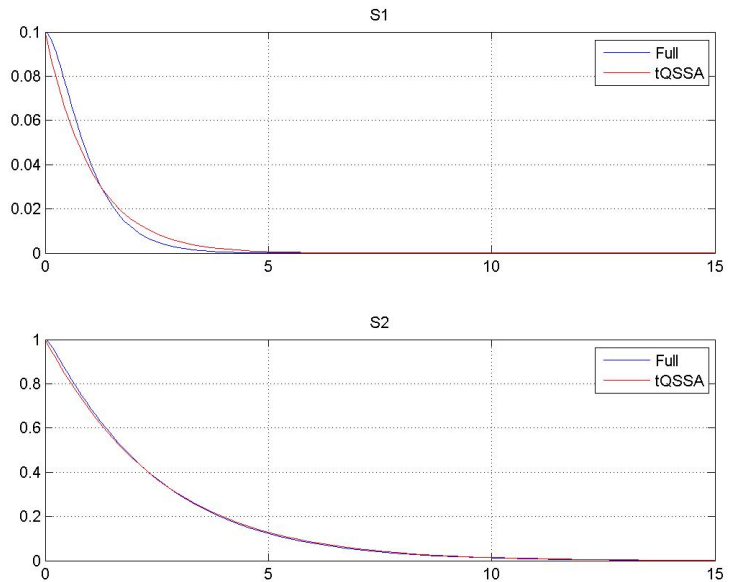

Figure 2. Comparison of the complexes (left) and of the substrates (right), solution of the system (35), with their tQSSA (36). The parameter set is the following: for the first reaction $a_{1}=k_{1}=1 ; d_{1}=0.1 ; E_{T}=1 ; S_{1} T=0.1 ; K_{1} M=1.1 ; K=1 ; \epsilon_{1}=0.23$; for the second reaction $a_{2}=k_{2}=1 ; d_{2}=3 ; E_{T}=1 ; S_{2} T=1 ; K_{2} M=4 ; K=1 ; \epsilon_{2}=0.03$. Thus $\epsilon=\epsilon_{1}=0.23$. Though the value of $\epsilon$ is very close to its bound $1 / 4$, the approximation is sufficiently satisfactory.

\section{The Center Manifold}

Let us start again from (33):

$$
\left\{\begin{aligned}
\frac{d \bar{s}_{1}}{d \tau} & =-\epsilon c_{1} \\
\frac{d c_{1}}{d \tau} & =-\left(\eta_{1}+\kappa_{1 M}\right) c_{1}+\bar{s}_{1}+\sigma_{1} \eta_{1} c_{1}^{2}+ \\
& -\bar{s}_{1}\left(\sigma_{2} c_{2}+\sigma_{1} c_{1}\right)+\sigma_{2} \eta_{1} c_{1} c_{2}, \\
\frac{d \bar{s}_{2}}{d \tau} & =-\epsilon c_{2} \frac{k_{2}}{k_{1}} \frac{\eta_{2}}{\eta_{1}} \\
\frac{d c_{2}}{d \tau} & =\frac{a_{2}}{a_{1}}\left[-\left(\eta_{1}+\frac{K_{2 M}}{K_{1 M}} \kappa_{1 M}\right) c_{2}+\frac{\eta_{1}}{\eta_{2}} \bar{s}_{2}\right]+ \\
& +\frac{a_{2}}{a_{1}}\left[\eta_{1} \sigma_{2} c_{2}^{2}-\sigma_{1} \frac{\eta_{1}}{\eta_{2}} \bar{s}_{2} c_{1}-\frac{S_{2 T}}{S_{1 T}} \sigma_{1} \bar{s}_{2} c_{2}+\sigma_{1} \eta_{1} c_{1} c_{2}\right] .
\end{aligned}\right.
$$


The origin $(0,0,0,0)$ is a fixed point of the system $(39)$.

We rewrite (39) in the form (14) and, to this aim, we operate the substitutions

$$
y_{1}=-\left(\eta_{1}+\kappa_{1 M}\right) c_{1}+\bar{s}_{1} \Leftrightarrow c_{1}=\frac{\bar{s}_{1}-y_{1}}{\eta_{1}+\kappa_{1 M}}
$$

and

$$
y_{2}=-\left(\eta_{1}+\frac{K_{2 M}}{K_{1 M}} \kappa_{1 M}\right) c_{2}+\frac{\eta_{1}}{\eta_{2}} \bar{s}_{2} \Leftrightarrow c_{2}=\frac{\frac{\eta_{1}}{\eta_{2}} \bar{s}_{2}-y_{2}}{\eta_{1}+\frac{K_{2 M}}{K_{1 M}} \kappa_{1 M}} .
$$

From (40) and (41) we have the new equations for $y_{1}$ and $y_{2}$ :

$$
\begin{aligned}
& \frac{d y_{1}}{d \tau}=\frac{d \bar{s}_{1}}{d \tau}-\left(\eta_{1}+\kappa_{1 M}\right) \frac{d c_{1}}{d \tau}=-\epsilon \frac{\bar{s}_{1}-y_{1}}{\eta_{1}+\kappa_{1 M}}+ \\
& -\left(\eta_{1}+\kappa_{1 M}\right)\left[y_{1}+\sigma_{1} \eta_{1}\left(\frac{\bar{s}_{1}-y_{1}}{\eta_{1}+\kappa_{1 M}}\right)^{2}-\bar{s}_{1}\left(\sigma_{2} \frac{\frac{\eta_{1}}{\eta_{2}} \bar{s}_{2}-y_{2}}{\eta_{1}+\frac{K_{2 M}}{K_{1 M}} \kappa_{1 M}}+\right.\right. \\
& \left.+\sigma_{1} \frac{\bar{s}_{1}-y_{1}}{\eta_{1}+\kappa_{1 M}}\right)+\sigma_{2} \eta_{1} \frac{\left(\bar{s}_{1}-y_{1}\right)\left(\frac{\eta_{1}}{\eta_{2}} \bar{s}_{2}-y_{2}\right)}{\left.\left(\eta_{1}+\kappa_{1 M}\right)\left(\eta_{1}+\frac{K_{2 M}}{K_{1 M}} \kappa_{1 M}\right)\right] .} \\
& \frac{d y_{2}}{d \tau}=\frac{\eta_{1}}{\eta_{2}} \frac{d \bar{s}_{2}}{d \tau}-\left(\eta_{1}+\frac{K_{2 M}}{K_{1 M}} \kappa_{1 M}\right) \frac{d c_{2}}{d \tau}=-\frac{k_{2}}{k_{1}} \epsilon \frac{\frac{\eta_{1}}{\eta_{2}} \bar{s}_{2}-y_{2}}{\eta_{1}+\frac{K_{2 M}}{K_{1 M}} \kappa_{1 M}}+ \\
& -\left(\eta_{1}+\frac{K_{2 M}}{K_{1 M}} \kappa_{1 M}\right)\left[\frac{a_{2}}{a_{1}} y_{2}+\frac{a_{2}}{a_{1}} \eta_{1} \sigma_{2}\left(\frac{\frac{\eta_{1}}{\eta_{2}} \bar{s}_{2}-y_{2}}{\left.\eta_{1}+\frac{K_{2 M}}{K_{1 M}} \kappa_{1 M}\right)^{2}+}\right.\right. \\
& -\frac{a_{2}}{a_{1}} \sigma_{1} \frac{\eta_{1}}{\eta_{2}} \bar{s}_{2} \frac{\bar{s}_{1}-y_{1}}{\eta_{1}+\kappa_{1 M}}-\frac{a_{2}}{a_{1}} \frac{S_{2 T}}{S_{1 T}} \sigma_{1} \bar{s}_{2} \frac{\frac{\eta_{1}}{\eta_{2}} \bar{s}_{2}-y_{2}}{\eta_{1}+\frac{K_{2 M}}{K_{1 M}} \kappa_{1 M}}+ \\
& \left.+\frac{a_{2}}{a_{1}} \sigma_{1} \eta_{1} \frac{\bar{s}_{1}-y_{1}}{\eta_{1}+\kappa_{1 M}} \frac{\frac{\eta_{1}}{\eta_{2}} \bar{s}_{2}-y_{2}}{\eta_{1}+\frac{K_{2 M}}{K_{1 M}} \kappa_{1 M}}\right] .
\end{aligned}
$$

By combining equations (42) and (43) with those in $\frac{d c_{1}}{d \tau}$ and $\frac{d c_{2}}{d \tau}-$ where $c_{1}, c_{2}$ are replaced by (40), (41) - and applying the technique described in $[8,9,21]$, the system $(39)$ becomes

$$
\left\{\begin{array}{l}
\frac{d \bar{s}_{1}}{d \tau}=-\epsilon \frac{\bar{s}_{1}-y_{1}}{\eta_{1}+\kappa_{1 M}}, \\
\frac{d \bar{s}_{2}}{d \tau}=-\frac{k_{2}}{k_{1}} \frac{\eta_{2}}{\eta_{1}} \epsilon \frac{\frac{\eta_{1}}{\eta_{2}} \bar{s}_{2}-y_{2}}{\eta_{1}+\frac{K_{2 M}}{K_{1 M}} \kappa_{1 M}}, \\
\frac{d y_{1}}{d \tau}=-\left(\eta_{1}+\kappa_{1 M}\right) y_{1}+f_{1}\left(\bar{s}_{1}, \bar{s}_{2}, y_{1}, y_{2}, \epsilon\right), \\
\frac{d y_{2}}{d \tau}=-\frac{a_{2}}{a_{1}}\left(\eta_{1}+\frac{K_{2 M}}{K_{1 M}} \kappa_{1 M}\right) y_{2}+f_{2}\left(\bar{s}_{1}, \bar{s}_{2}, y_{1}, y_{2}, \epsilon\right), \\
\frac{d \epsilon}{d \tau}=0
\end{array}\right.
$$




\section{Tihonov theory in inhibitory reactions.}

where we have isolated the linear part in $\left(\bar{s}_{1}, \bar{s}_{2}, y_{1}, y_{2}, \epsilon\right)$ and

$$
\begin{aligned}
& f_{1}\left(\bar{s}_{1}, \bar{s}_{2}, y_{1}, y_{2}, \epsilon\right):=-\epsilon \frac{\bar{s}_{1}-y_{1}}{\eta_{1}+\kappa_{1 M}}-\sigma_{1} \eta_{1} \frac{\left(\bar{s}_{1}-y_{1}\right)^{2}}{\eta_{1}+\kappa_{1 M}}+ \\
& +\left(\eta_{1}+\kappa_{1 M}\right) \sigma_{2} \frac{\bar{s}_{1}\left(\frac{\eta_{1}}{\eta_{2}} \bar{s}_{2}-y_{2}\right)}{\eta_{1}+\frac{K_{2 M}}{K_{1 M}} \kappa_{1 M}}+\sigma_{1} \bar{s}_{1}\left(\bar{s}_{1}-y_{1}\right)+ \\
& -\sigma_{2} \eta_{1} \frac{\left(\bar{s}_{1}-y_{1}\right)\left(\frac{\eta_{1}}{\eta_{2}} \bar{s}_{2}-y_{2}\right)}{\eta_{1}+\frac{K_{2 M}}{K_{1 M}} \kappa_{1 M}},
\end{aligned}
$$

and

$$
\begin{aligned}
& f_{2}\left(\bar{s}_{1}, \bar{s}_{2}, y_{1}, y_{2}, \epsilon\right):=-\frac{k_{2}}{k_{1}} \epsilon \frac{\frac{\eta_{1}}{\eta_{2}} \bar{s}_{2}-y_{2}}{\eta_{1}+\frac{K_{2 M}}{K_{1 M}} \kappa_{1 M}}-\frac{a_{2}}{a_{1}} \eta_{1} \sigma_{2} \frac{\left(\frac{\eta_{1}}{\eta_{2}} \bar{s}_{2}-y_{2}\right)^{2}}{\eta_{1}+\frac{K_{2 M}}{K_{1 M}} \kappa_{1 M}}+ \\
& +\left(\eta_{1}+\frac{K_{2 M}}{K_{1 M}} \kappa_{1 M}\right) \frac{a_{2}}{a_{1}} \sigma_{1} \frac{\eta_{1}}{\eta_{2}} \frac{\bar{s}_{2}\left(\bar{s}_{1}-y_{1}\right)}{\eta_{1}+\kappa_{1 M}}+ \\
& +\frac{a_{2}}{a_{1}} \frac{S_{2 T}}{S_{1 T}} \sigma_{1} \bar{s}_{2}\left(\frac{\eta_{1}}{\eta_{2}} \bar{s}_{2}-y_{2}\right)-\frac{a_{2}}{a_{1}} \sigma_{1} \eta_{1} \frac{\left(\bar{s}_{1}-y_{1}\right)\left(\frac{\eta_{1}}{\eta_{2}} \bar{s}_{2}-y_{2}\right)}{\eta_{1}+\kappa_{1 M}}
\end{aligned}
$$

(From here on out we will adopt the notation $\frac{d y}{d \tau}=\dot{y}$.)

Considering the equilibrium point $(0,0,0,0,0)$, the associated linearized system has a diagonal form and, moreover, the eigenvalues are given by 0 (with multiplicity $c=3$ ) and

$$
\lambda_{1}=-\left(\eta_{1}+\kappa_{1 M}\right), \quad \lambda_{2}=-\frac{a_{2}}{a_{1}}\left(\eta_{1}+\frac{K_{2 M}}{K_{1 M}} \kappa_{1 M}\right) .
$$

All the eigenvalues are $\leq 0$. To find a center manifold, all we need to do is to solve (16) for system (44), employing Theorem 2.2, which gives us a method for computing an approximate solution of (16) to any desired degree of accuracy. So we search for a function $h(x, \epsilon)$ - where $h: \mathbb{R}^{3} \rightarrow \mathbb{R}^{2}$ and $x=\left(\bar{s}_{1}, \bar{s}_{2}\right)-$ such that

$$
h(x, \epsilon)=\left(\begin{array}{l}
h_{1}(x, \epsilon) \\
h_{2}(x, \epsilon)
\end{array}\right)
$$

and

$$
\begin{aligned}
& h_{1}\left(\bar{s}_{1}, \bar{s}_{2}, \epsilon\right)=\alpha_{1} \bar{s}_{1}^{2}+\alpha_{2} \bar{s}_{2}^{2}+\alpha_{3} \epsilon^{2}+\alpha_{4} \bar{s}_{1} \bar{s}_{2}+\alpha_{5} \bar{s}_{1} \epsilon+\alpha_{6} \bar{s}_{2} \epsilon+\ldots \\
& h_{2}\left(\bar{s}_{1}, \bar{s}_{2}, \epsilon\right)=\beta_{1} \bar{s}_{1}^{2}+\beta_{2} \bar{s}_{2}^{2}+\beta_{3} \epsilon^{2}+\beta_{4} \bar{s}_{1} \bar{s}_{2}+\beta_{5} \bar{s}_{1} \epsilon+\beta_{6} \bar{s}_{2} \epsilon+\ldots
\end{aligned}
$$




\section{Bersani, Borri, Milanesi, Vellucci}

Hence, by Definition 2.3, the center manifold is:

$$
F:=\left(\begin{array}{l}
y_{1}(x, \epsilon) \\
y_{2}(x, \epsilon)
\end{array}\right)=\left(\begin{array}{l}
h_{1}(x, \epsilon) \\
h_{2}(x, \epsilon)
\end{array}\right)
$$

Let $\xi=\left(\bar{s}_{1}, \bar{s}_{2}, \epsilon\right)$, we have that, from (48), (49) and (50):

$$
\begin{aligned}
& f_{1}\left(\bar{s}_{1}, \bar{s}_{2}, \epsilon\right)=-\frac{\bar{s}_{1} \epsilon}{\eta_{1}+\kappa_{1 M}}-\frac{\sigma_{1} \eta_{1} \bar{s}_{1}^{2}}{\eta_{1}+\kappa_{1 M}}+ \\
& +\frac{\frac{\eta_{1}}{\eta_{2}} \sigma_{2}\left(\eta_{1}+\kappa_{1 M}\right) \bar{s}_{1} \bar{s}_{2}}{\eta_{1}+\frac{K_{2 M}}{K_{1 M}} \kappa_{1 M}}+\sigma_{1} \bar{s}_{1}^{2}-\frac{\frac{\eta_{1}^{2}}{\eta_{2}} \sigma_{2} \bar{s}_{1} \bar{s}_{2}}{\eta_{1}+\frac{K_{2 M}}{K_{1 M}} \kappa_{1 M}}+o\left(|\xi|^{2}\right),
\end{aligned}
$$

$$
\begin{aligned}
& f_{2}\left(\bar{s}_{1}, \bar{s}_{2}, \epsilon\right)=-\frac{\frac{k_{2}}{k_{1}} \frac{\eta_{1}}{\eta_{2}} \bar{s}_{2} \epsilon}{\eta_{1}+\frac{K_{2 M}}{K_{1 M}} \kappa_{1 M}}-\frac{\frac{a_{2}}{a_{1}} \frac{\eta_{1}^{3}}{\eta_{2}^{2}} \sigma_{2} \bar{s}_{2}^{2}}{\eta_{1}+\frac{K_{2 M}}{K_{1 M}} \kappa_{1 M}}+ \\
& +\left(\eta_{1}+\frac{K_{2 M}}{K_{1 M}} \kappa_{1 M}\right) \frac{a_{2}}{a_{1}} \frac{\eta_{1}}{\eta_{2}} \sigma_{1} \frac{\bar{s}_{1} \bar{s}_{2}}{\eta_{1}+\kappa_{1 M}}+\frac{a_{2}}{a_{1}} \frac{\eta_{1}}{\eta_{2}} \frac{S_{2 T}}{S_{1 T}} \sigma_{1} \bar{s}_{2}^{2}+ \\
& -\frac{a_{2}}{a_{1}} \sigma_{1} \frac{\frac{\eta_{1}^{2}}{\eta_{2}} \bar{s}_{1} \bar{s}_{2}}{\eta_{1}+\kappa_{1 M}}+o\left(|\xi|^{2}\right) .
\end{aligned}
$$

for $\xi \rightarrow 0$.

By definition of center manifold and from (47), we calculate

$$
\dot{y}_{1}=D_{x} h_{1}(x, \epsilon) \dot{x}+D_{\epsilon} h_{1}(x, \epsilon) \underbrace{\dot{\epsilon}}_{=0}=\lambda_{1} y_{1}+f_{1}\left(x, y_{1}, y_{2}\right)
$$

and

$$
\dot{y}_{2}=D_{x} h_{2}(x, \epsilon) \dot{x}+D_{\epsilon} h_{2}(x, \epsilon) \underbrace{\dot{\epsilon}}_{=0}=\lambda_{2} y_{2}+f_{2}\left(x, y_{1}, y_{2}\right),
$$

which are a consequence of (16).

We start from (53). It can be shown that

$$
D_{x} h_{1}(x, \epsilon) \dot{x}=\frac{\partial h_{1}}{\partial \bar{s}_{1}} \dot{\overline{s_{1}}}+\frac{\partial h_{1}}{\partial \bar{s}_{2}} \dot{\overline{s_{2}}}=o\left(|\xi|^{2}\right)
$$

for $\xi \rightarrow 0$, then (53) becomes

$$
\lambda_{1} y_{1}+f_{1}\left(x, y_{1}, y_{2}\right)=o\left(|\xi|^{2}\right)
$$




\section{Tihonov theory in inhibitory reactions.}

or, from (48), (49),(50) and more explicitly:

$$
\begin{aligned}
& -\left(\eta_{1}+\kappa_{1 M}\right)\left(\alpha_{1} \bar{s}_{1}^{2}+\alpha_{2} \bar{s}_{2}^{2}+\alpha_{3} \epsilon^{2}+\alpha_{4} \bar{s}_{1} \bar{s}_{2}+\alpha_{5} \bar{s}_{1} \epsilon+\right. \\
& \left.+\alpha_{6} \bar{s}_{2} \epsilon+o\left(|\xi|^{2}\right)\right)-\frac{\bar{s}_{1} \epsilon}{\eta_{1}+\kappa_{1 M}}-\frac{\sigma_{1} \eta_{1} \bar{s}_{1}^{2}}{\eta_{1}+\kappa_{1 M}}+ \\
& +\frac{\frac{\eta_{1}}{\eta_{2}} \sigma_{2}\left(\eta_{1}+\kappa_{1 M}\right) \bar{s}_{1} \bar{s}_{2}}{\eta_{1}+\frac{K_{2 M}}{K_{1 M}} \kappa_{1 M}}+\sigma_{1} \bar{s}_{1}^{2}-\frac{\frac{\eta_{1}^{2}}{\eta_{2}} \sigma_{2} \bar{s}_{1} \bar{s}_{2}}{\eta_{1}+\frac{K_{2 M}}{K_{1 M}} \kappa_{1 M}}+o\left(|\xi|^{2}\right)=o\left(|\xi|^{2}\right) .
\end{aligned}
$$

Equating to zero terms of the same powers gives $\alpha_{2}=\alpha_{3}=\alpha_{6}=0$ and:

$$
\begin{gathered}
-\left(\eta_{1}+\kappa_{1 M}\right) \alpha_{1}-\frac{\sigma_{1} \eta_{1}}{\eta_{1}+\kappa_{1 M}}+\sigma_{1}=0 \\
-\left(\eta_{1}+\kappa_{1 M}\right) \alpha_{4}+\frac{\frac{\eta_{1}}{\eta_{2}} \sigma_{2}\left(\eta_{1}+\kappa_{1 M}\right)}{\eta_{1}+\frac{K_{2 M}}{K_{1 M}} \kappa_{1 M}}-\frac{\frac{\eta_{1}^{2}}{\eta_{2}} \sigma_{2}}{\eta_{1}+\frac{K_{2 M}}{K_{1 M}} \kappa_{1 M}}=0 \\
\left(\eta_{1}+\kappa_{1 M}\right) \alpha_{5}+\frac{1}{\eta_{1}+\kappa_{1 M}}=0,
\end{gathered}
$$

from which

$(56)$

$$
\alpha_{1}=\frac{\kappa_{1 M} \sigma_{1}}{\left(\eta_{1}+\kappa_{1 M}\right)^{2}} \quad ; \quad \alpha_{4}=\frac{\frac{\eta_{1}}{\eta_{2}} \sigma_{2} \kappa_{1 M}}{\left(\eta_{1}+\kappa_{1 M}\right)\left(\eta_{1}+\frac{K_{2 M}}{K_{1 M}} \kappa_{1 M}\right)} \quad ; \quad \alpha_{5}=-\frac{1}{\left(\eta_{1}+\kappa_{1 M}\right)^{2}} .
$$

Similar argumentations can be used to handle (54). At the end, we obtain the center manifold of (44)

$$
\begin{aligned}
& y_{1}\left(\bar{s}_{1}, \bar{s}_{2}, \epsilon\right)=h_{1}\left(\bar{s}_{1}, \bar{s}_{2}, \epsilon\right)=\frac{\kappa_{1 M} \sigma_{1}}{\left(\eta_{1}+\kappa_{1 M}\right)^{2}} \bar{s}_{1}^{2}+ \\
& +\frac{\frac{\eta_{1}}{\eta_{2}} \sigma_{2} \kappa_{1 M}}{\left(\eta_{1}+\kappa_{1 M}\right)\left(\eta_{1}+\frac{K_{2 M}}{K_{1 M}} \kappa_{1 M}\right)} \bar{s}_{1} \bar{s}_{2}-\frac{1}{\left(\eta_{1}+\kappa_{1 M}\right)^{2}} \bar{s}_{1} \epsilon+o\left(|\xi|^{2}\right) .
\end{aligned}
$$

$$
\begin{aligned}
& y_{2}\left(\bar{s}_{1}, \bar{s}_{2}, \epsilon\right)=h_{2}\left(\bar{s}_{1}, \bar{s}_{2}, \epsilon\right)=\frac{\eta_{1}}{\eta_{2}} \frac{\frac{S_{2 T}}{S_{1 T}} \sigma_{1}\left(\eta_{1}+\frac{K_{2 M}}{K_{1 M}} \kappa_{1 M}\right)-\frac{\eta_{1}^{2}}{\eta_{2}} \sigma_{2}}{\left(\eta_{1}+\frac{K_{2 M}}{K_{1 M}} \kappa_{1 M}\right)^{2}} \bar{s}_{2}^{2}+ \\
& +\frac{\frac{\eta_{1}}{\eta_{2}} \sigma_{1} \frac{K_{2 M}}{K_{1 M}} \kappa_{1 M}}{\left(\eta_{1}+\frac{K_{2 M}}{K_{1 M}} \kappa_{1 M}\right)\left(\eta_{1}+\kappa_{1 M}\right)} \bar{s}_{1} \bar{s}_{2}-\frac{\frac{a_{2}}{a_{1}} \frac{\eta_{1}}{\eta_{2}}}{\left(\eta_{1}+\frac{K_{2 M}}{K_{1 M}} \kappa_{1 M}\right)^{2}} \bar{s}_{2} \epsilon+o\left(|\xi|^{2}\right) .
\end{aligned}
$$


Bersani, Borri, Milanesi, Vellucci

Figures 3 and 4 show the behavior of the center manifold and of the trajectories of the solutions and their tQSSAs in the phase space $\left(\left(\bar{s}_{1}, \bar{s}_{2}, c_{1}\right)\right.$ on the left and $\left(\bar{s}_{1}, \bar{s}_{2}, c_{2}\right)$ on the right), for $\epsilon=0.23$. In Figure 4 we have rotated the center manifold, in order to show more clearly the asymptotic equivalence of the manifold and of the trajectories. While in the plots on the left we can observe that the trajectories of the points $\left(\bar{s}_{1}, \bar{s}_{2}, c_{1}\right)$ are asymptotically equivalent to the center manifold, as expected from the theory, in the plots on the right the comparison is absolutely unsatisfactory.

In [16] it was shown that for a single enzyme reaction the tQSSA is asymptotically equivalent to the center manifold of the system. For more complex reactions we still lack a mathematical justification of this fact which, however, seems to be a reasonable result, confirmed by the plots on the left.

We can however explain the apparently contradictory behavior in the plots on the right with the fact that in this case the value of $\epsilon$ is not sufficiently small to guarantee the applicability of the theory.

Presumably, the computation of corrections of higher order in $\epsilon$ could improve our results.

\section{Conclusion and perspectives}

In this paper we have applied Palsson theory related to the separation of time scales in linear enzyme reactions to the case of the inhibition mechanism. We were able to state sufficient conditions for the separation of variables in this case, too, determining an appropriate perturbation parameter $\epsilon$, which becomes crucial for the application of Tihonov's Theorem. We have also explicitly computed the center manifold for this kind of systems and compared it with the trajectories of the solutions and of their tQSSAs. We observed that, even for high values of $\epsilon$, the tQSSA well approximates the solution of the differential system governing the reactions. We related this fact to the consideration that the tQSSA can be seen as the application, under suitable hypotheses, of Tihonov theory for differential systems characterized by the presence of small parameters. As already observed in other papers (see, for example, $[1,4-6]$ ), any QSSA can be interpreted as the leading order term of an asymptotic expansion of the solutions with respect of an appropriate parameter. The unsatisfactory behavior of the center manifold and of the tQSSA with respect to the solution of the differential system can be corrected determining the successive terms at order $\epsilon$. This will be subject of a future work. We will also study the bifurcation analysis of the differential system associated to the inhibition mechanism.

Palsson theory seems very promising in order to handle more complex 


\section{Tihonov theory in inhibitory reactions.}
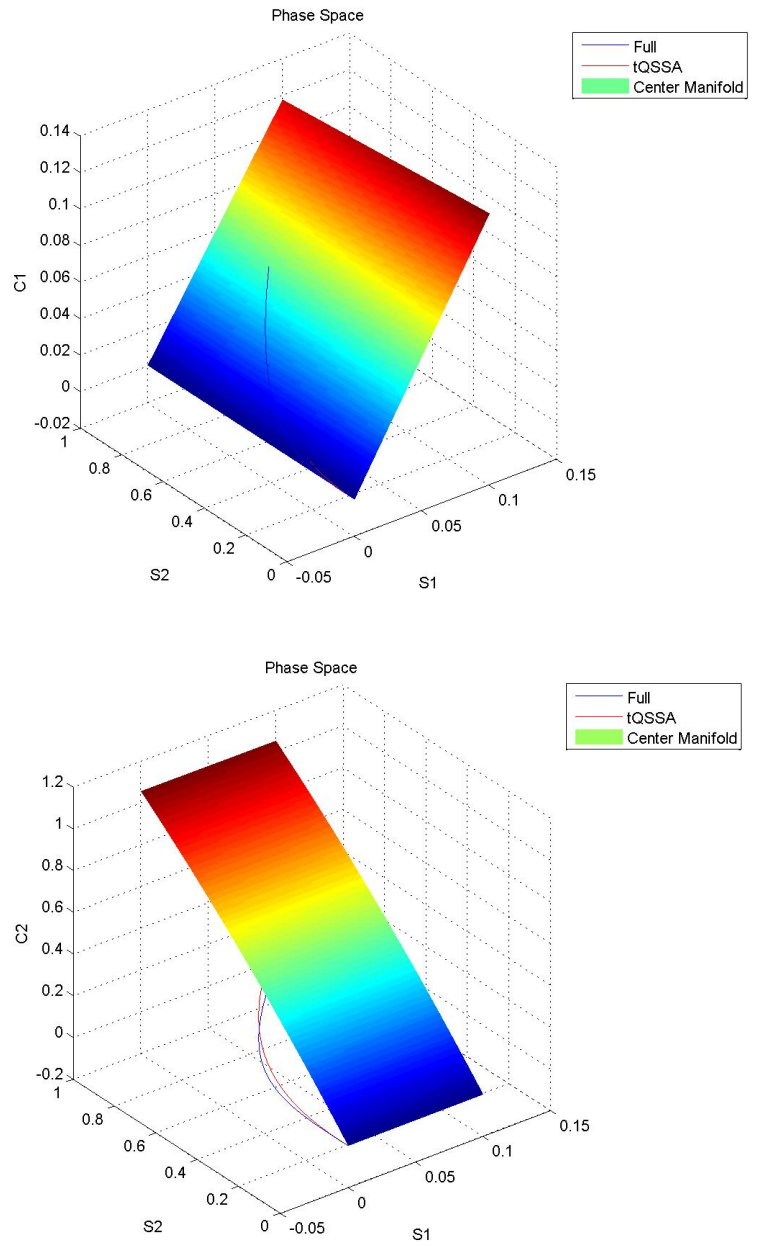

Figure 3. Comparison among the behavior of the center manifold, the trajectory of the solutions of the system (24) and of their tQSSAs for $\left(\bar{s}_{1}, \bar{s}_{2}, c_{1}\right)$ (left) and $\left(\bar{s}_{1}, \bar{s}_{2}, c_{2}\right)$ (right), in the stressed case $\epsilon=0.23$. The center manifold is here clearly shown.

mechanisms by means of Tihonov theory and Center Manifold Theory. In a work in preparation, we are trying to apply these techniques to several mechanisms of phosphorylation and dephosphorylation, including cycles of single and double phosphorylation.

\section{Acknowledgements}

The authors are deeply grateful to Prof. Enzo Orsingher, from Sapienza University (Rome, Italy) and Prof. Jan Andres, from Palacky University 
Bersani, Borri, Milanesi, Vellucci
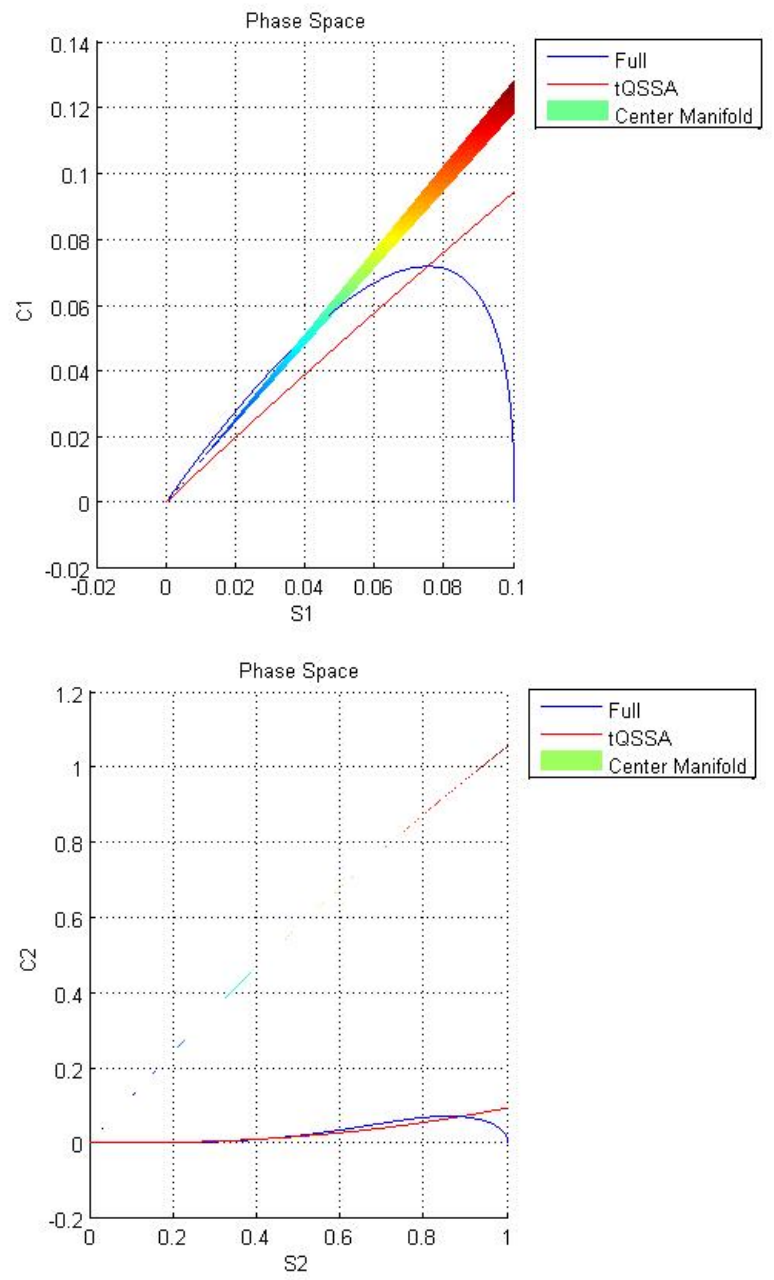

Figure 4. Comparison among the behavior of the center manifold, the trajectory of the solutions of the system (24) and of their tQSSAs for $\left(\bar{s}_{1}, \bar{s}_{2}, c_{1}\right)$ (left) and $\left(\bar{s}_{1}, \bar{s}_{2}, c_{2}\right)$ (right), in the stressed case $\epsilon=0.23$. The plots are here rotated in order to show more clearly the trajectories. While in the plot on the left we can appreciate the convergence of the trajectories to the center manifold, in the plot on the right there are evident differences, which can be explained with the high value of the parameter $\epsilon$.

(Olomouc, Czech Republic) for their translations of the papers [10-12] and some precious clarifications concerning some passages of the papers. 
Tihonov theory in inhibitory reactions.

\section{Bibliography.}

\section{REFERENCES}

1. J. Murray, Mathematical Biology: An introduction. Springer-Verlag New York, 2002.

2. J. Borghans, R. de Boer, and L. Segel, Extending the quasi-steady state approximation by changing variables, Bull.Math.Biol., vol. 58, pp. 4363, 1996.

3. A. Bersani, E. Bersani, G. Dell'Acqua, and M. Pedersen, New trends and perspectives in nonlinear intracellular dynamics: one century from michaelis-menten paper, CMAT, vol. 27, pp. 659-684, 2015.

4. F. G. Heineken, T. M., and A. R., On the mathematical status of the pseudo-steady state hypothesis of biochemical kinetics, Math. Biosc., vol. 1, pp. 95-11, 1967.

5. L. A. Segel and M. Slemrod, The quasi steady-state assumption: a case study in pertubation., Siam Rev., vol. 31, pp. 446-477, 1989.

6. G. Dell'Acqua and A. M. Bersani, A perturbation solution of michaelismenten kinetics in a "total" framework, Journal of Mathematical Chemistry, vol. 50, no. 5, pp. 1136-1148, 2012.

7. J. Carr, Applications of Center Manifold Theory. Springer-Verlag New York, Heidelberg, Berlin, 1981.

8. S. Wiggins, Normally Hyperbolic Invariant Manifolds in Dynamical Systems, vol. 105. Springer-Verlag New York, 1994.

9. S. Wiggins, Introduction to applied nonlinear dynamical systems and chaos, vol. 2. Springer-Verlag New York, 2003.

10. A. Tikhonov, On the dependence of the solutions of differential equations on a small parameter, Mat. Sb. (N.S.), vol. 22, no. 2, pp. $193-$ 204, 1948.

11. A. Tikhonov, On a system of differential equations containing parameters, Mat. Sb. (N.S.), vol. 27, pp. 147-156, 1950.

12. A. Tikhonov, Systems of differential equations containing small parameters in the derivatives, Mat. Sb. (N.S.), vol. 31, no. 3, pp. 575-586, 1952.

13. W. Wasov, Asymptotic Expansions for Ordinary Differential Equations. Wiley-InterScience, 1965.

14. I. Dvořák and J. Ššska, Analysis of metabolic systems with complex slow and fast dynamics, Bulletin of Mathematical Biology, vol. 51, no. 2, pp. 255-274, 1989.

15. A. Kumar and K. Josić, Reduced models of networks of coupled enzymatic reactions, Journal of Theoretical Biology, vol. 278, no. 1, pp. 87 
Bersani, Borri, Milanesi, Vellucci

$-106,2011$.

16. A. Bersani, E. Bersani, A. Borri, and P. Vellucci, "Dynamical aspects of the total QSSA in enzyme kinematics." https://arxiv.org/abs/1702. 05351. Submitted to Journal of Mathematical Biology.

17. B. O. Palsson and E. N. Lightfoot, Mathematical modelling of dynamics and control in metabolic networks. i. on michaelis-menten kinetics, Journal of Theoretical Biology, vol. 111, no. 2, pp. 273 - 302, 1984.

18. B. O. Palsson, R. Jamier, and E. N. Lightfoot, Mathematical modelling of dynamics and control in metabolic networks. ii. simple dimeric enzymes, Journal of Theoretical Biology, vol. 111, no. 2, pp. 303 - 321, 1984.

19. B. O. Palsson, H. Palsson, and E. N. Lightfoot, Mathematical modelling of dynamics and control in metabolic networks. iii. linear reaction sequences, Journal of Theoretical Biology, vol. 113, no. 2, pp. 231 - 259, 1985.

20. B. O. Palsson, On the dynamics of the irreversible michaelis-menten reaction mechanism, Chemical Engineering Science, vol. 42, no. 3, pp. 447 - 458, 1987.

21. A. J. Roberts, Model emergent dynamics in complex systems. SIAM, 2015. 